\title{
Supporting tools for automated timetable planning
}

\author{
N. Bešinović, E. Quaglietta \& R. M. P. Goverde \\ Department of Transport and Planning, \\ Delft University of Technology, The Netherlands
}

\begin{abstract}
To satisfy the growing demand in railway transportation, infrastructure managers have the necessity to design more effective timetables. To this aim it is necessary to rely on automatic timetabling support tools that can provide feasible timetables with improved performance. In this paper we propose a hierarchical framework for timetable design that includes a microscopic, mesoscopic and macroscopic model of the network. These three models interact with each other in a closed-loop in order to generate an optimal timetable that is feasible at the level of track detection sections. An iterative adjustment of train running and minimum headway times is performed in the framework which stops when a feasible timetable is generated. Different from the other approaches in literature, this framework always guarantees timetable feasibility. Additional timetable performance is also realized in terms of stability, robustness, and energy efficiency. The application to an area of the Dutch railway network shows the ability of the framework in checking the feasibility of a timetable and evaluating its stability by determining the corresponding capacity occupation. In this sense practitioners can use this framework either for effective timetabling and postevaluation of existing timetables.
\end{abstract}

Keywords: timetable, feasibility, robustness, stability, capacity analysis.

\section{Introduction}

The recent growth in the demand for railway passengers and freight has raised the necessity for infrastructure managers to improve the efficiency of their networks in terms of increased capacity, higher service availability (e.g. punctuality, regularity) and lower energy consumption. Upgrading the 
infrastructure (e.g. allocating new tracks) or the signalling and ATP systems (e.g. installing ETCS level 2) is a suitable solution to achieve these objectives but are very costly to implement. A cost-effective alternative is represented by an effective planning of train services to get timetables that are able to absorb stochastic disturbances during operations while exploiting network capacity as much as possible. This means allocating as many train paths as possible to the available infrastructure slots while guaranteeing time allowances (i.e. supplements and buffer times) that effectively dampen delay propagation. In this context timetable design must rely on a very accurate estimation of the infrastructure slots to reserve for the allocation of train paths. Only a precise definition of these slots allows the effective design of dense timetables that are operationally feasible, i.e., free from conflicts and respecting the constraints imposed by the infrastructure layout, the signalling/ATP, and the interlocking systems. Macroscopic models that are used so far by infrastructure managers for designing railway timetables must be substituted or integrated with more detailed models that ensure the operational feasibility of the timetable. To this purpose different approaches have been proposed in literature based on a hierarchical integration of timetabling models with different levels of detail. Schlechte et al. [1] present a bottom-up approach that first aggregates microscopic running and headway times in order to be used by a macroscopic model that identifies the optimal timetable for a given utility function. Then the feasibility of this timetable is verified by simulating it at microscopic level and checking that there are not overlaps of blocking times among trains. Middelkoop [2] developed the tool ROBERTO that computes accurate headway times based on a microscopic infrastructure model which are used as input to the macroscopic timetabling model DONS [3]. De Fabris et al. [4] introduce a mesoscopic timetabling model with a simplified representation of station layouts that allows the combining of fast computation of macroscopic models with the accuracy of microscopic models. The main shortcomings with these approaches are that some of them (e.g. $[2,4])$ do not perform any feasibility check of the timetable produced, while others (e.g. [1]) do not consider any iterative modification to the timetable if this is proved to be unfeasible at microscopic level.

In order to overcome such limitations, this paper proposes a hierarchical framework for the optimal design of railway timetables in which a microscopic, mesoscopic and macroscopic model interact by iteratively adapting microscopic running and headway times until the produced macroscopic timetable is proved feasible. Differently from the other approaches, additional functions are available that deal with stability, robustness and energy efficiency of the timetable.

The main advantages of this framework are: $i$ ) the guarantee that the timetables obtained are always operationally feasible and $i$ ) the possibility of interchanging functions and models of the framework with other models with the same level of detail, without changing the structure of the communication process.

The proposed approach has been applied in a case study consisting of a part of the Dutch network that includes the corridors Utrecht-Den Bosch-Eindhoven and Tilburg-Nijmegen. 
Sections 2 and 3 describe the network modelling and the structure of the framework while sections 4,5 and 6 give detailed information on the inputoutput and the functions used in the micro, meso and macro model respectively. Section 7 illustrates the application to the case study and the corresponding results. Conclusions and future research are reported in Section 8 .

\section{A hierarchical network modelling}

The hierarchical framework for timetable design is composed of three models that respectively represent the same network with a microscopic, mesoscopic and a macroscopic level of detail (Figure 1).
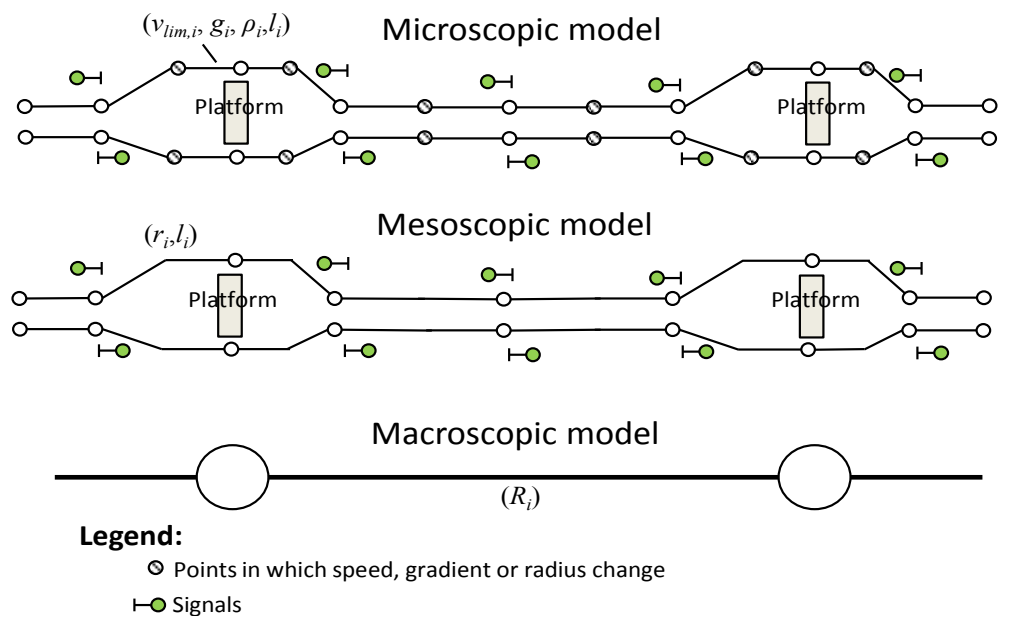

Figure 1: Infrastructure representation of the microscopic, mesoscopic and macroscopic models.

The microscopic model represents the network at the level of homogeneous behavioural sections (i.e. sections with constant values of speed limit, gradient and curvature radius). This means that this model is a graph having as arcs infrastructure sections with constant characteristics of speed limit $v_{\text {lim }}$, gradient $g$ and radius $\rho$. The set of attributes $\left(v_{\text {lim }, i}, g_{i}, \rho_{i}, l_{i}\right)$ is associated to arc $i$, having length $l_{i}$. Microscopic nodes represent either points in which these characteristics change, and infrastructure elements like signals, switches, platforms, etc. The mesoscopic model aggregates the homogeneous behavioural sections in block sections for open tracks, and track-free detection sections for interlocking areas. With this kind of aggregation it is possible to consider sectional route release within interlocking areas. The $i$-th arc of such a model has two attributes $\left(r_{i}, l_{i}\right)$, namely the running time $r_{i}$ on the arc (as computed by the micro model) and its length $l_{i}$. The nodes represent both the joints between consecutive block or track detection sections and infrastructure elements such as signals, switches, platforms, etc. The macroscopic model aggregates mesoscopic 
arcs into corridors between two consecutive stations or junctions. The $i$-th arc of this model depicts the corridor between two successive stations or junctions and its attribute $R_{i}$ is the corridor running time as aggregated from the micro model. The nodes represent here the stations and the junctions of the network.

\section{Algorithmic framework}

The structure of the proposed hierarchical framework for timetable design is shown in Figure 2, which indicates the interactions among the different models, their functions as well as the input-output data flow. The framework has a standardized RailML interface that consents to load input data from RailML format. In particular, the RailML data necessary for the initialization of the framework relate to characteristics of the infrastructure (i.e. gradients, speed limits, positions of stations, switches), rolling stock (i.e. mass, composition, braking rate, tractive effort-speed curve), interlocking (i.e. alternative local routes), signalling system (e.g. position and type of signals), and routes/stopping pattern of the train services to be scheduled. These data are transformed by a RailML converter tool into a series of ascii files having a specific format readable by the microscopic model. In this way the microscopic model is initialized and all the other models are consequently built-up. At this point, the micro model computes minimum running times Min $R T$ considering timetable design parameters like turn-around times and minimum dwell times at stations. If a macroscopic timetable (Macro TT) has not been computed yet, operational running times (Oper. $R T$ ) are calculated by adding the minimum amount of supplement times (e.g. 5\% of the running time) to the Min RT. Once a Macro TT has been produced, the operational running times are computed by respecting the time allowances (supplements and buffer times) and the arrival/departure times given by the timetable. In this case the function Operational Running Time computation can also calculate the operational running times by identifying train time-distance trajectories that exploit timetable supplement times to minimize the energy consumption. The operational running times are then set as input to the mesoscopic model that estimates blocking times for every train. If no Macro TT exists yet, blocking times are sent to the function Min Headway computation to compute minimum headways. Otherwise blocking times are sent to the conflict detection module that verifies the feasibility of the timetable. If there are no overlaps of blocking times, the timetable is feasible and it is transferred to the Capacity Evaluation function that marks up the timetable as stable if the amount of supplement times respects the thresholds established by the UIC norms [9].

If instead the blocking times of consecutive trains overlap, the timetable is not feasible and new headway times must be computed that allow non-conflicting train services. The function Min Headway computation is therefore activated both when no Macro TT is available yet, and when the Macro TT is infeasible.

These minimum headways are then transferred together with the minimum running times (from the micro model) to the macroscopic model. The latter computes a new Macro TT by means of mathematical algorithms that optimize a given objective function (e.g. maximizing throughput or minimizing waiting 


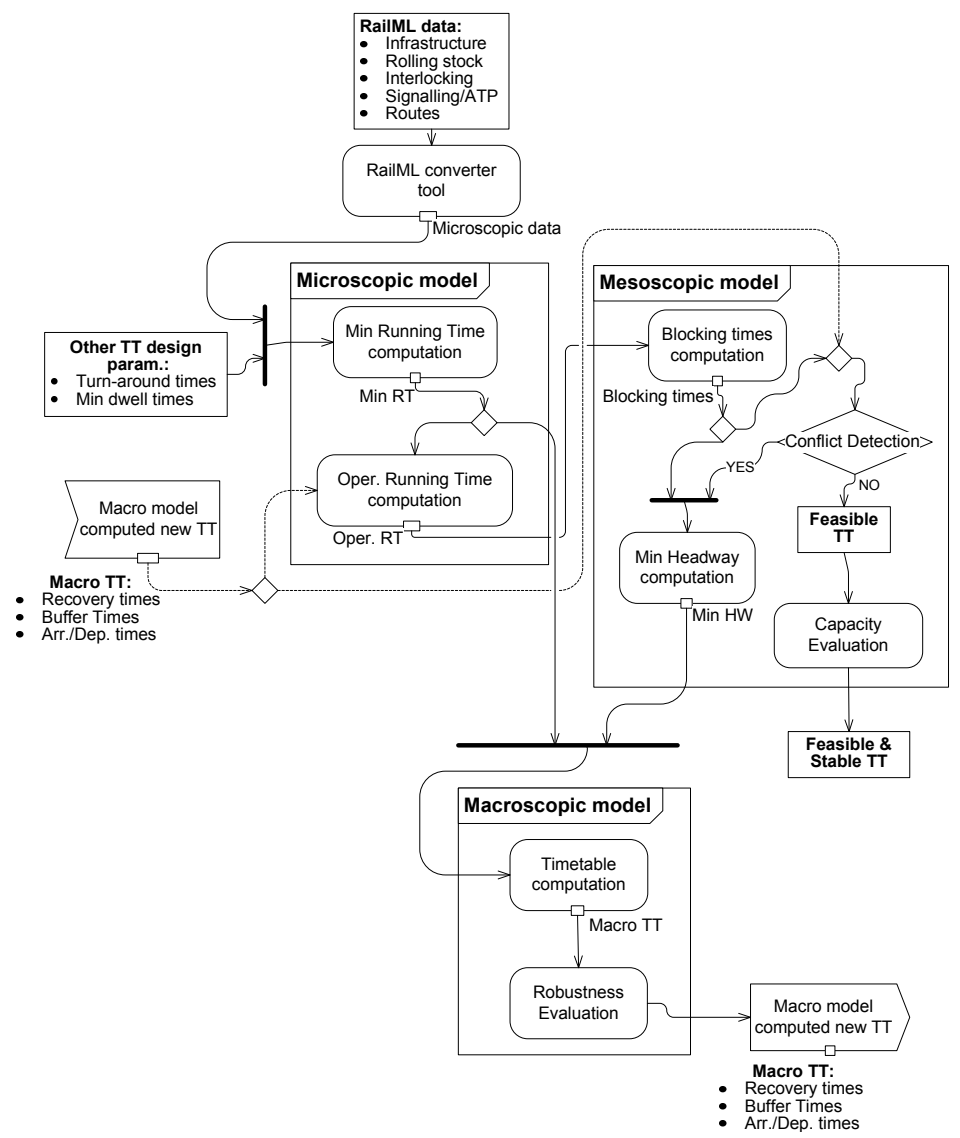

Figure 2: SysML scheme of the hierarchical framework for timetable design.

time). Once this timetable is obtained, it is evaluated on robustness by estimating its ability of absorbing stochastic disturbances to operations. If the timetable has an acceptable level of robustness, an event is triggered that activates the microscopic model by sending the Macro TT. At this point, the microscopic model computes again the operational running times which are sent to the mesoscopic model that in turn elaborates blocking times and verifies the feasibility of the timetable. This loop continues until the timetable produced by the macroscopic model is proved to be feasible. The final output of the framework is therefore a feasible, stable and robust timetable with improved energy-efficient train trajectories.

\section{Microscopic model}

The input data of the microscopic model are: i) characteristics of the infrastructure, the rolling stock, the signalling/ATP and interlocking systems, as 
well as train routes; $i$ ) Timetable design parameters like minimum dwell times at stations and turn-around times; iii) arrival/departure times and time allowances given by the macroscopic timetable.

The output data are: i) minimum running times, i.e., the free-flow running time of a train assuming that it runs at the maximum allowed speeds; and ii) operational running times, i.e., the running time scheduled in the timetable that is adopted during operations.

The functions applied in the microscopic model are the following:

a) Minimum running time computation. This function computes minimum train running times taking into account all the physical characteristics of the infrastructure, the rolling stock the signalling/ATP and the interlocking systems. Running times are calculated by numerical integration of the Newton's motion formula. The tractive effort is assumed as a piecewise function of speed consisting of linear and hyperbolic parts. Train resistances are modelled by the Davis equation [6], a second-order polynomial of speed. A constant braking rate is considered for braking phases. More details about this model can be found in [5].

b) Operational running time computation. Operational running times are those scheduled in the timetable which are computed by adding to the minimum running time a supplement time (e.g. 5\%) to make up for statistical variations of running times during real operations. This function is activated when a macroscopic timetable has been already produced. In particular, this function calculates time-distance and speed-distance trajectories assuming that trains exploit the whole supplement times fixed by the macroscopic timetable. Two options can be used to exploit supplement times: $i$ ) cruising with a speed lower than the maximum, or ii) introducing energy efficient driving strategies such as coasting, cruising at optimal speeds and reducing the amount of acceleration/braking phases. For the latter option, energyefficient train trajectories are computed by applying optimal control algorithms. Figure 3 shows the speed-time trajectories computed by the micro model relative to the minimum running time (part $a$ ) and the operational running time that uses an energy-efficient coasting phase (part $b)$.
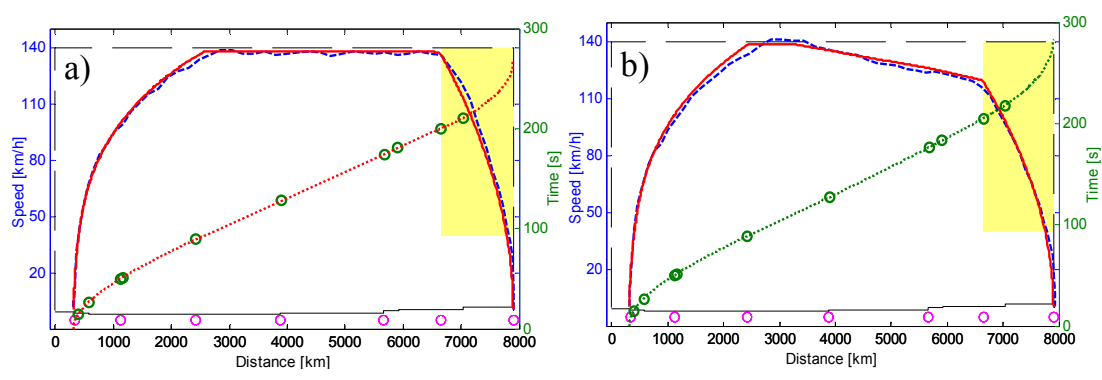

Figure 3: Speed-distance diagrams: a) minimum running time, b) optimal energy-efficient trajectory. 


\section{Mesoscopic model}

The input data of the mesoscopic model are represented by: $i$ ) minimum running times, $i$ ) operational running times, and iii) arrival/departure times scheduled by the macroscopic timetable.

The output data consist of: $i$ ) blocking time stairs, $i i$ ) minimum headways for each couple of consecutive trains, and iii) the infrastructure occupation rate.

The functions applied for the mesoscopic model are the following:

a) Blocking times computation. Blocking times (Figures $4 \mathrm{~b}$ and c) are computed by applying the procedure to build up blocking times as described in [6]. If a macroscopic timetable is not available yet, blocking times are computed considering the distance-time trajectories that give the minimum running times. Otherwise blocking times are calculated based on the operational distance-time trajectories as given by the timetable.

b) Conflict Detection. This function is activated only when a macroscopic timetable has been produced already. The aim is to verify the feasibility of the macroscopic timetable by checking the absence of track conflicts. Track conflicts are detected as overlaps of the blocking times provided by the Blocking Time Computation function. If the timetable is conflict-free it is evaluated in terms of capacity consumption and stability, otherwise it is necessary to compute new minimum headways that allow conflict-free train operations.

c) Min Headway Computation. For every station and junction of the network (i.e. for every node of the macro model) minimum headway times are computed for each pair of consecutive trains. Specifically, a set of minimum headways is calculated for all the possible cases of interactions between two consecutive trains: e.g. both trains leaving a station, both trains entering a station or one entering and the other leaving. For each one of these cases minimum headways are calculated by applying the UIC compression method where trains are shifted in order that their blocking time stairs are touching but not overlapping. The compression and the computation of the minimum headways are performed by using the method proposed by Egmond [7] that is based on max-plus algebra. Figure 4d shows an example how this method works to compute the minimum headway time between two trains A and B passing through the same station. These two trains are scheduled to depart at the same time but they have different routes: sections 1, 2, 3, and 5 for train $\mathrm{A}$, and sections 4, 3, 2, and 1 for train $\mathrm{B}$. The minimum headway between these trains is obtained by shifting the departure of one train (in this case train B) in order that their blocking time stairs are just touching. The minimum headway for the station is then identified as the distance between the original departure time of train A (that has not been shifted) and the shifted departure time of train B.

Capacity Evaluation. Feasible macroscopic timetables are evaluated by this function in terms of capacity as established by the UIC code 406. In particular the infrastructure occupation is evaluated, defined as the minimum time needed to operate trains according to a given timetable pattern. To 


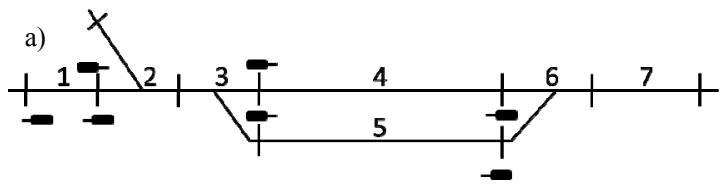

Routes:

A: 1-2-3-5

B: $4-3-2-1$

C: $2-3-4$
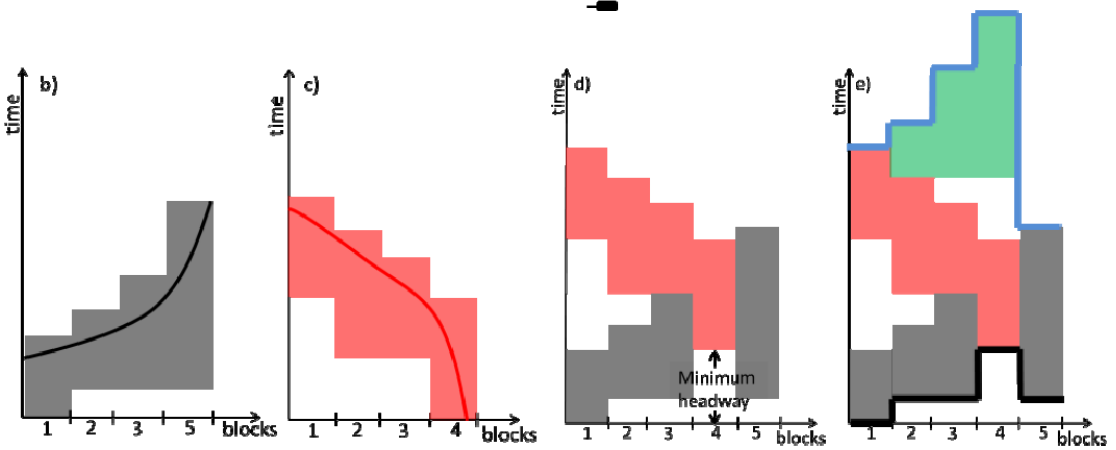

Figure 4: Infrastructure example with blocking times of trains A (a) and B (b), minimum headway time computation (c), and infrastructure occupation for schedule $\mathrm{ABC}(\mathrm{d})$.

determine this minimum time it is necessary to compress all train paths scheduled in a given time period. This compression is performed by using again the method proposed by Egmond. For example if in one hour the timetable schedules three trains A, B and C (Figure 4e), the capacity occupation is obtained by compressing all trains. The white spaces left (in between the blue and black contour lines) represent the lost capacity. After computing the capacity consumption, the buffer time can be determined. The larger the time buffer time the better the ability of the timetable to prevent initial and secondary delays, i.e., the timetable is more stable. If the amount of buffer time is higher than the amount recommended by the UIC code 406, the timetable is considered as stable. Otherwise it is defined unstable and operational running times must be recomputed by e.g. adding additional supplement and buffer times.

\section{Macroscopic model}

The input data of the macroscopic model are: $i$ ) minimum running times from the micro model aggregated by corridors; and ii) minimum headway times for stations and junctions relative to each pair of trains for all the possible cases of interaction.

The output of this model is the macroscopic timetable giving scheduled departure/arrival times at stations for each train, passing times trough junctions and time allowances.

The functions adopted in the macroscopic model are described as follows:

a) Timetable computation. This function uses exact optimization techniques or heuristics to construct a timetable that optimizes a given objective function. 
Such a function is completely interchangeable meaning that any kind of macroscopic timetabling model can be adopted. For example this function can be performed by optimization models for constructing periodic timetables (e.g. $[3,10]$ ) or by models suitable for non-periodic timetables (e.g. [11]). Independently from the optimization model used the output of this function will be an optimized macroscopic timetable.

b) Robustness Evaluation. Once the macroscopic timetable is produced (by the previous function), this is simulated against a set of disturbed scenarios to measure its ability in mitigating the propagation of delays. A timetable is defined as robust if it is able to mitigate the delay propagation on the network when stochastic disturbances to operations occur. To this purpose trains are simulated according to the timetable considering stochastic perturbations to their running times. A specific cost function of robustness is defined to measure the rate of the introduced perturbations that has been absorbed by the timetable. The value of this cost function gives a measure of how the timetable is robust towards stochastic disturbances.

\section{Case study}

The hierarchical framework proposed has been tested in a real case study: an area of the Dutch railway network that includes the two intersecting corridors Utrecht-Eindhoven and Tilburg-Nijmegen. The microscopic model of this network is represented by a graph having 2000 nodes and 3000 arcs. When aggregating this graph by block sections (on open lines) and track detection sections (in interlocking areas) we obtain the corresponding mesoscopic representation constituted by a graph with 800 nodes and 1000 arcs. The corresponding macroscopic model is instead composed of 15 nodes and 28 arcs. A periodic timetable, with an hour period, is currently in operation over the considered area. The hourly timetable pattern contains 5 Intercity (IC) and 5 regional trains per direction between Utrecht and Eindhoven; 3 IC and 3 regional trains per direction between Tilburg and Njimegen; and 2 ICs and 2 regional trains per direction between Eindhoven and Tilburg. A total of 40 train runs per hour are therefore scheduled over the whole area. Freight trains are here neglected. For the sake of clarity we will focus on the corridor with the densest traffic, that is to say the Utrecht-Eindhoven corridor. For brevity the aim of our application is not to build up a new macroscopic timetable from scratch, but to analyse the timetable currently in operation on such a corridor.

This timetable was designed with the support of the macroscopic timetabling tool DONS, that in this case would represent the macroscopic model in our hierarchical framework. Specifically the analysis considers one period of the timetable, that is to say one full hour. This timetable is imported to the microscopic model which by means of the function Operational running time computation estimates the operational train trajectories. In detail such trajectories are computed by considering trains running with a lower cruising speed to exploit the running time supplements in the timetable. The operational timedistance trajectories are then transferred to the mesoscopic model that estimates 


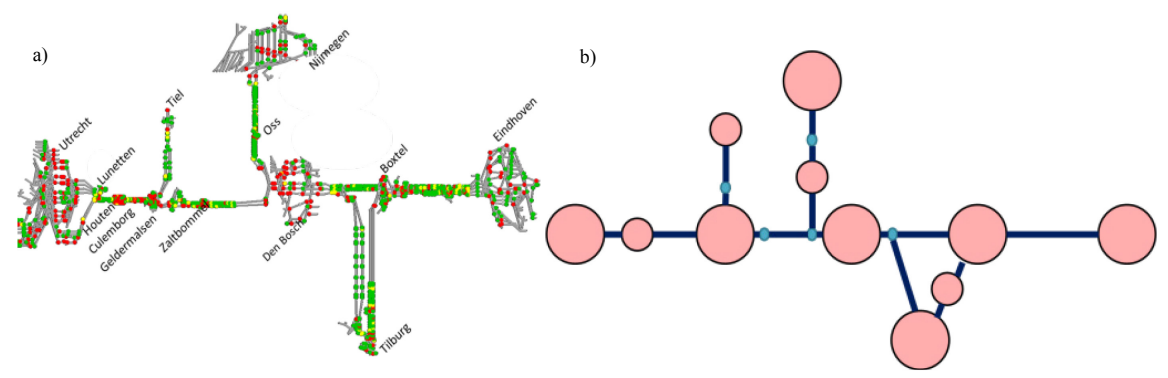

Figure 5: a) Graphical representation and b) macrocscopic network of corridor.

train blocking times needed by the Conflict Detection function. This latter function has identified that the timetable considered on this corridor does not have overlaps of train blocking times, hence it is feasible. Given that it is feasible a capacity evaluation is performed to estimate its performances in terms of infrastructure occupation and stability. Graphical results of this evaluation are shown in Figure 6 where we report the infrastructure occupation relative to the station of Geldermalsen. The arcs of the macroscopic model for this station coincide with track detection sections since Geldermalsen is an interlocking area. The blocking times in Figure 6 are therefore depicted for each track detection sections. Here block sections are computed for minimum running times without considering additional running time supplements. The aim is to identify the whole amount of time allowances allocated by the timetable, including the running time supplements, the buffer times and blank time slots left for running potential freight trains. Each colour corresponds to a different train service passing through the station. All the track detection sections that are used by trains are enumerated and reported on the x-axis. This numbering does not have any geographical meaning but is only an identification number. The diagram immediately shows the adoption of sectional route release in this station. By applying the method proposed by Egmond to compress blocking time stairs, we obtain that the whole infrastructure occupation in Geldermalsen is $758 \mathrm{~s}$, i.e., 12.6 minutes. This means that the timetable contains locally around $47.4 \mathrm{~min}(78 \%)$ of unused capacity for including running time supplements, buffer times and blank time slots for freight trains. According to the norms provided by the UIC code 406 the station of Geldermalsen has an acceptable amount of time margins, resulting in a good local timetable stability Each colour determines one train movement, i.e., a train arriving, dwelling and departing or passing through the station. Analysing the outcome of Capacity evaluation, one can observe: $a$ ) each train adjoins a preceding and a following one by at least one block. b) Track detection section 9 represents a platform, with longer corresponding blocking times, and $c$ ) sectional route releases. Note that the last train is just the repeated first train (red) from the next period. This one is needed as a reference to determine the infrastructure occupation rate [9]. Based on the last, the occupation rate (double arrow) equals 758 s, i.e., $12.6 \mathrm{~min}$. Therefore, if 
no overtaking was scheduled; we would have at most 47.4 min for distributing time allowances to increase the timetable performances and/or to schedule additional trains.
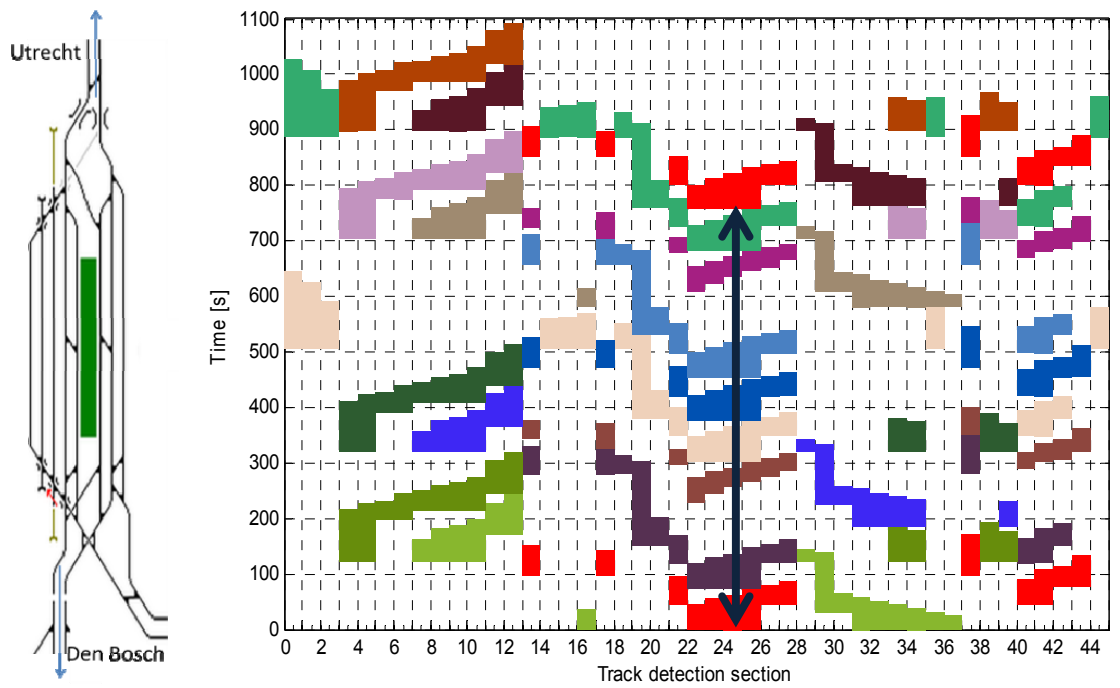

Figure 6: Station Geldermalsen: a) track layout, b) capacity occupation.

\section{Conclusions}

This paper introduced an innovative hierarchical framework for supporting the design of railway timetables. A microscopic, mesoscopic and macroscopic model of the same network interact with each other in order to produce a feasible macroscopic timetable. Differently from the other multi-level timetabling models proposed in literature, this framework always guarantees that the timetable provided is feasible. This is realized by a closed-loop interaction between the three models that in case of an infeasible timetable modifies iteratively running and minimum headway times until the feasibility check is successful. Moreover, the timetable is evaluated on energy-efficiency, robustness and infrastructure occupation which gives insights on the stability. The practical application to an area of the Dutch railway network shows the ability of this framework in identifying the feasibility of macroscopic timetables at the level of track detection sections. Furthermore it is illustrated that by estimating the infrastructure occupation we can obtain information on the total amount of allowance time used in the timetable, i.e., its capability in absorbing delays (i.e. the level of stability). Practitioners and timetable designers can use this framework both for timetable design and evaluation of existing timetables. Future research will be addressed to generate and evaluate timetables that also include short-term freight train path requests. A specific study will be dedicated to define performance measures that evaluate the resilience of the timetable, i.e., 
the ability of restoring scheduled operations when real-time rescheduling is applied during perturbed traffic.

\section{Acknowledgements}

This research is funded by the European FP7 project Optimal Networks for Train Integration Management across Europe (ON-TIME) under Grant Agreement number FP7-SCP0-GA-2011-265647. We thank the Dutch infrastructure manager ProRail and the Netherlands Railways (NS) for the data provided.

\section{References}

[1] Schlechte T., Borndorfer R., Erol B., Graffagnino T., Swarat E., Micromacro transformation of railway networks, Journal of Rail Transport Planning and Management, Vol. 1, pp. 38-48, 2011.

[2] Middlekoop, A.D., Headway generation with ROBERTO, WIT Transactions on the built environment, Vol. 114, WIT Press, 2010, pp. 431-439.

[3] Kroon L., Huisman D., Abbink E., Fioole P.J., Fischetti M., Maroti G., Schrijver A., Steenbeek A., Ybema R., The New Dutch Timetable: The OR revolution, Interfaces, Vol. 39 (1), pp. 6-17, 2009.

[4] De Fabris S., Longo G., Medeossi G., Pesenti R., Application and validation of a timetabling algorithm to a large Italian network, Proceedings of the $5^{\text {th }}$ International Seminar on Railway Operations Modelling and Analysis (RailCopenhagen), Copenhagen, 13-15 May, 2013.

[5] Besinovic N., Quaglietta E., Goverde R.M.P., A simulation-based optimization approach for the calibration of dynamic train speed profiles, Journal of Rail Transport Planning and Management, in press, 2013.

[6] Hansen I.A., Pachl J., Railway Timetable and Traffic, Eurailpress, Hamburg, Germany, 2008.

[7] Egmond R.J., An algebraic approach for scheduling train movements, The 8th International Conference on Computer-Aided Scheduling of Public Transport (CASPT 2000), Berlin, 21-23 June, 2000.

[8] Goverde, R.M.P., Railway Timetable Stability Analysis Using Max-Plus Sys-tem Theory. Transportation Research Part B, 41(2), 179-201, 2007.

[9] UIC Code 406: Capacity. International Union of Railways, Paris, $2^{\text {nd }}$ Edition, June 2012.

[10] Sparing D., Goverde R.M.P., An Optimization Model for Periodic Timetable Generation with Dynamic Frequencies, 16th International IEEE Conference on Intelligent Transport Systems (IEEE ITSC 2013), 6-9 October, The Hague, 2013.

[11] Cacchiani, V., Caprara, A., Fischetti, M., A Lagrangian Heuristic for Robustness, with an Application to Train Timetabling, Transportation Science, Vol. 46(1), pp. 124-133, 2012. 